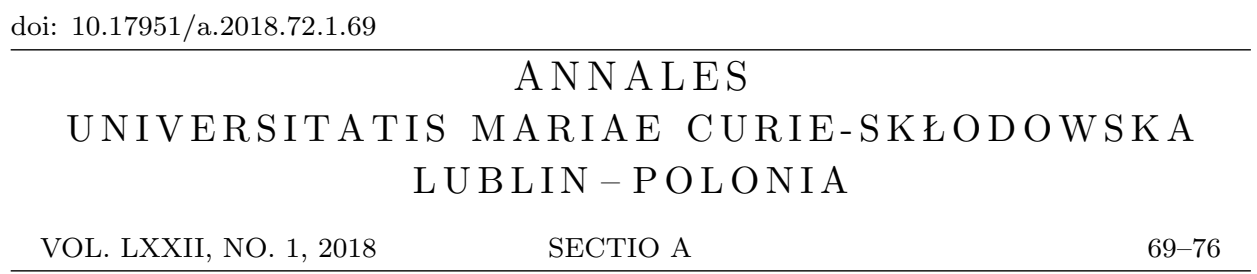

PAWE€ OCHALIK and ANDRZEJ WŁOCH

\title{
On generalized Mersenne numbers, their interpretations and matrix generators
}

\begin{abstract}
In this paper we introduce generalized Mersenne numbers. We shall present some of their interpretations and matrix generators which are very useful for determining identities.
\end{abstract}

1. Introduction and preliminary results. Let $k \geq 3$ be a fixed integer. For any integer $n \geq 0$ let $M(k, n)$ be the $n$th generalized Mersenne number defined by the second order linear recurrence relation of the form

$$
M(k, n)=k M(k, n-1)-(k-1) M(k, n-2)
$$

for $n \geq 2$ with initial conditions $M(k, 0)=0$ and $M(k, 1)=1$. Then generalized Mersenne numbers form the generalized Mersenne sequence $0,1, k$, $k^{2}-k+1, \ldots$

If $k=3$, then $M(3, n)$ is the well-known $n$th Mersenne number and it is denoted by $M_{n}$. Moreover, $M_{n}=2^{n}-1$ and consequently the Mersenne sequence has the form $0,1,3,7,15, \ldots$ The Mersenne numbers are discussed mainly with the concept of Mersenne prime. Moreover, the Mersenne number is well known as the solution of the famous problem of the Hanoi tower.

The Mersenne numbers, their generalizations, properties and relations with the numbers of the Fibonacci type were studied in many papers, see for instance [8], [9], [11].

2010 Mathematics Subject Classification. 11B37, 15B36.

Key words and phrases. Mersenne numbers, Fibonacci numbers, matrix generators. 
In this paper we study generalized Mersenne numbers $M(k, n)$, their properties, matrix generators and some combinatorial interpretations.

Theorem 1. Let $n \geq 0, k \geq 3$ be integers. Then

$$
M(k, n)=\frac{1}{k-2}\left((k-1)^{n}-1\right) .
$$

Proof. If $n=0,1$, then the equality holds from the definition of $M(k, n)$. Let $n \geq 2$ and let us consider the recurrence

$$
M(k, n)=k M(k, n-1)-(k-1) M(k, n-2) .
$$

Then solving the characteristic equation of the form $r^{n}-k r^{n-1}+$ $(k-1) r^{n-2}=0$ and using initial conditions $M(k, 0)=1$ and $M(k, 1)=k$, the result immediately follows.

Corollary 2. Let $n \geq 1, k \geq 3$ be integers. Then $M(k, n+1)=\sum_{i=0}^{n-1}(k-1)^{i}$.

Proof. It immediately follows from Theorem 1 and the fact that $\frac{1}{k-2}\left((k-1)^{n}-1\right)$ is the sum of $n$ first terms of the geometric sequence.

The equality $M(k, n)=\frac{1}{k-2}\left((k-1)^{n}-1\right)$ is a Binet-type formula for generalized Mersenne numbers $M(k, n)$.

Theorem 3. Let $k \geq 3, n \geq 1, p \geq 1$ be integers. Then

(a) $M(k, n)=M(k, p+1) M(k, n-p)-(k-1) M(k, p) M(k, n-p-1)$,

(b) $M(k, n)=(k-1)^{p} M(k, n-p)+M(k, p)$,

(c) $M(k, n+1)-M(k, n)=(k-1)^{n}$.

Proof. (a) (By induction on $p$ ). If $p=1$, then $M(k, 2) M(k, n-1)-$ $(k-1) M(k, 1) M(k, n-2)=k M(k, n-1)-(k-1) M(k, n-2)=M(k, n)$ by the definition of the generalized Mersenne numbers. Assume that for an arbitrary $p>1$ the formula $M(k, n)=M(k, p+1) M(k, n-p)-(k-$ 1) $M(k, p) M(k, n-p-1)$ holds. We shall show that the equality is true for $p+1$. Using the induction hypothesis and the definition of $M(k, n)$, we obtain

$$
\begin{aligned}
& M(k, n)=M(k, p+1) M(k, n-p)-(k-1) M(k, p) M(k, n-p-1) \\
& =M(k, p+1)(k M(k, n-p-1)-(k-1) M(k, n-p-2)) \\
& \quad-(k-1) M(k, p) M(k, n-p-1) \\
& =M(k, n-p-1)(k M(k, p+1)-(k-1) M(k, p)) \\
& \quad-(k-1) M(k, p+1) M(k, n-p-2) \\
& =M(k, n-p-1) M(k, p+2)-(k-1) M(k, p+1) M(k, n-p-2) \\
& =M(k, n-(p+1)) M(k, p+2) \\
& \quad-(k-1) M(k, p+1) M(k, n-(p+1)-1),
\end{aligned}
$$


which ends the proof.

(b) Let $p=1$. We will show that $M(k, n)=(k-1) M(k, n-1)+1$. By solving the equation $M(k, n)=(k-1) M(k, n-1)+1$, being nonhomogeneous first order linear recurrence equation, we obtain $M(k, n)=\frac{1}{k-2}\left((k-1)^{n}-1\right)$ and by the Theorem 1 the result follows.

Let $p \geq 2$. Then using the above relation for $M(k, n)$, we have

$$
\begin{aligned}
M(k, n) & =(k-1) M(k, n-1)+1=(k-1)[(k-1) M(k, n-2)+1]+1 \\
& =(k-1)^{2} M(k, n-2)+1+(k-1) \\
& =(k-1)^{2} M(k, n-2)+M(k, 2) .
\end{aligned}
$$

Repeating it $p$ times, $p \geq 2$, we obtain $M(k, n)=(k-1)^{p} M(k, n-p)+$ $1+(k-1)+(k-1)^{2}+(k-1)^{3}+\cdots+(k-1)^{p-1}$.

Using the Corollary 2 we have that $M(k, n)=(k-1)^{p} M(k, n-p)+M(k, p)$, which ends the proof.

(c) Immediately follows from Corollary 2.

From the above we obtain identities for the classical Mersenne numbers.

Corollary 4. Let $n \geq 1, p \geq 1$ be integers. Then

(a) $M_{n}=M_{p+1} M_{n-p}-2 M_{p} M_{n-p-1}$,

(b) $M_{n}=2^{p} M_{n-p}+M_{p}$,

(c) $M_{n+1}-M_{n}=2^{n}$.

2. Matrix generators. Matrix methods are important in studying recurrence relations. In the last decades some mathematicians have found and studied miscellaneous affinities between matrices and linear recurrences. Using matrix methods, different identities and algebraic representations of considered sequences can be obtained, see for instance [3], [5], [6], [10].

In recurrence relations the Fibonacci numbers play an important role and they are studied intensively also with the context of matrix generators. We recall that the Fibonacci numbers are defined by the recurrence $F_{n}=F_{n-1}+$ $F_{n-2}$ for $n \geq 2$ with $F_{0}=F_{1}=1$. The wide theory of the classical Fibonacci numbers includes a lot of identities and using some of them, we can show the relations between generalized Mersenne numbers and the classical Fibonacci numbers. As an example, we can write that $M(k, n)-(k-1) M(k, n-1)=$ $F_{n+1}-\sum_{i=0}^{n-1} F_{i}$.

Interesting relations between $(s, t)$-Fibonacci numbers and Mersenne numbers were given by Civciv and Turkmen in [2]. Relations between the Fibonacci numbers and other numbers of the Fibonacci type were studied for instance in [3], [10]. In this section we study the matrix generators for the generalized Mersenne numbers, also using the Hessenberg matrix. For the classical Fibonacci sequence, the matrix generator, named 
the golden matrix, has the form $Q=\left[\begin{array}{ll}1 & 1 \\ 1 & 0\end{array}\right]$. It is well known that $Q^{n}=\left[\begin{array}{cc}F_{n} & F_{n-1} \\ F_{n-1} & F_{n-2}\end{array}\right]$, for $n \geq 2$

In this section we give some matrix generators for generalized Mersenne numbers $M(k, n)$.

Theorem 5. Let $n \geq 1, k \geq 3$ be integers. Then

$$
\left[\begin{array}{cc}
M(k, n+2) & M(k, n+1) \\
M(k, n+1) & M(k, n)
\end{array}\right]=\left[\begin{array}{cc}
k & 1 \\
1 & 0
\end{array}\right]\left[\begin{array}{cc}
k & 1 \\
-(k-1) & 0
\end{array}\right]^{n} .
$$

Proof. (By induction on $n$ ). If $n=1$, then the result is obvious. Suppose that the equality holds for an arbitrary $n, n \geq 2$. We shall show that it is true for $n+1$. Using the induction hypothesis, we have

$$
\begin{aligned}
{\left[\begin{array}{ll}
k & 1 \\
1 & 0
\end{array}\right] } & {\left[\begin{array}{cc}
k & 1 \\
-(k-1) & 0
\end{array}\right]^{n+1} } \\
& =\left[\begin{array}{ll}
k & 1 \\
1 & 0
\end{array}\right]\left[\begin{array}{cc}
k & 1 \\
-(k-1) & 0
\end{array}\right]^{n}\left[\begin{array}{cc}
k & 1 \\
-(k-1) & 0
\end{array}\right] \\
& =\left[\begin{array}{cc}
M(k, n+2) & M(k, n+1) \\
M(k, n+1) & M(k, n)
\end{array}\right]\left[\begin{array}{cc}
k & 1 \\
-(k-1) & 0
\end{array}\right] \\
& =\left[\begin{array}{cc}
k M(k, n+2)-(k-1) M(k, n+1) & M(k, n+2) \\
k M(k, n+1)-(k-1) M(k, n) & M(k, n+1)
\end{array}\right] \\
& =\left[\begin{array}{cc}
M(k, n+3) & M(k, n+2) \\
M(k, n+2) & M(k, n+1)
\end{array}\right]
\end{aligned}
$$

by the recurrence definition of the generalized Mersenne numbers.

From Theorem 5 and basic properties of matrices we have

Corollary 6. Let $n \geq 1, k \geq 3$ be integers. Then

(a) $M(k, n+2) M(k, n)-M^{2}(k, n+1)=-(k-1)^{n}$,

(b) $\left[\begin{array}{c}M(k, n+1) \\ M(k, n)\end{array}\right]=\left[\begin{array}{cc}k & -(k-1) \\ 1 & 0\end{array}\right]^{n}\left[\begin{array}{l}1 \\ 0\end{array}\right]$.

The identity given in (a) will be named the Cassini formula for the generalized Mersenne numbers. If $k=3$, then $M_{n+2} M_{n}-M_{n+1}^{2}=-2^{n}$ is the Cassini formula for the classical Mersenne numbers.

A square matrix $H_{n}=\left[h_{i j}\right]$ of size $n$ is the lower Hessenberg matrix if $h_{i j}=0$ for $j-i>1$. Hessenberg matrices are used as matrix generators of numbers of the Fibonacci type, see [7], [4]. In this paper we define a Hessenberg matrix as the generator of the generalized Mersenne numbers. 
Let $H_{n}$ be the lower Hessenberg matrix of the size $n$ of the form

$$
H_{n}=\left[\begin{array}{cccccc}
k & 1 & 0 & \ldots & 0 & 0 \\
k-1 & k & 1 & \ldots & 0 & 0 \\
0 & k-1 & k & \ldots & 0 & 0 \\
\vdots & \vdots & \vdots & \vdots & \vdots & \vdots \\
0 & \ldots & \ldots & \ldots & k & 1 \\
0 & \ldots & \ldots & \ldots & k-1 & k
\end{array}\right] .
$$

In other words, $h_{i i}=k, h_{i, i+1}=1, h_{i-1, i}=k-1$ for $i=1, \ldots, n$ and $h_{i j}=0$ in the remaining cases.

Theorem 7. Let $n \geq 1, k \geq 3$ be integers. Then $\operatorname{det} H_{n}=M(k, n+1)$.

Proof. If $n=1$, then $\operatorname{det} H_{n}=\operatorname{det}[k]=k=M(k, 2)$. If $n=2$, then $\operatorname{det} H_{2}=\operatorname{det}\left[\begin{array}{cc}k & 1 \\ k-1 & k\end{array}\right]=k^{2}-k+1=M(k, 3)$. Let $n \geq 3$. Suppose that $\operatorname{det} H_{t}=M(k, t+1)$ for $t \leq n$. We shall show that $\operatorname{det} H_{n+1}=M(k, n+2)$. By fundamental properties of determinants we obtain

$$
\begin{aligned}
& \operatorname{det} H_{n+1}=\left|\begin{array}{cccccc}
k & 1 & 0 & \ldots & 0 & 0 \\
k-1 & k & 1 & \ldots & 0 & 0 \\
0 & k-1 & k & \ldots & 0 & 0 \\
\vdots & \vdots & \vdots & \vdots & \vdots & \vdots \\
0 & \ldots & \ldots & \ldots & k & 1 \\
0 & \ldots & \ldots & \ldots & k-1 & k
\end{array}\right| \\
& =k \cdot \operatorname{det} H_{n}-(k-1)\left|\begin{array}{ccccc}
1 & 0 & \ldots & 0 & 0 \\
k-1 & k & \ldots & 0 & 0 \\
\vdots & \vdots & \vdots & \vdots & \vdots \\
0 & 0 & \ldots & k & 1 \\
0 & 0 & \ldots & k-1 & k
\end{array}\right| \\
& =k \cdot \operatorname{det} H_{n}-(k-1) \operatorname{det} H_{n-1} \text {. }
\end{aligned}
$$

Using the induction hypothesis and the recurrence definition of the Mersenne numbers, we obtain det $H_{n+1}=k \cdot M(k, n+1)-(k-1) M(k, n)=M(k, n+2)$, which ends the proof.

3. Interpretations of the generalized Mersenne numbers. Combinatorial interpretations of numbers given by the recurrence relations play an important role in finding their distinct properties and were studied in [1], [10].

In this section we give some interpretations of the generalized Mersenne numbers. Let us consider the alphabet of $k$ letters, $k \geq 3$ of the form $\mathcal{A}=\left\{A_{1}, \ldots, A_{k}\right\}$. For $n \geq 1$ we consider the word $a_{1} a_{2} \ldots a_{n}$ of the length $n$ created from letters $A_{1}, \ldots, A_{k}$ such that 
(i) $a_{i} \neq a_{i+1}, i=1, \ldots, n-1$, or

(ii) $a_{j}=A_{1}$ for all $j=1, \ldots, p, 1 \leq p \leq n$ and $a_{i} \neq a_{i+1}$, for all $p+1 \leq i \leq$ $n-1$

Let $\sigma(k, n)$ denote the number of all words $a_{1} \ldots a_{n}$ satisfying conditions (i) and (ii).

Theorem 8. Let $n \geq 1, k \geq 3$ be integers. Then

$$
\sigma(k, n)=\frac{1}{k-2}\left((k-1)^{n+1}-1\right) .
$$

Proof. Let $A_{1}, \ldots, A_{k}$ be given letters, for $k \geq 3$. Let $n \geq 1$. We construct the words of the length $n$. If $n=1$, then we have $k$ distinct words, so $\sigma(k, 1)=\frac{1}{k-2}\left((k-1)^{2}-1\right)=k$. In the next step for $n=2$, we have $k$ possible words with the initial letter $A_{1}$ and $(k-1)$ possibilities for $(k-1)$ remaining cases. Consequently, we obtain $k+(k-1)^{2}=1+k(k-1)$ distinct words. Words with the initial letter $A_{1}$ have the form $A_{1} A_{1}, A_{1} A_{2}, \ldots, A_{1} A_{k}$. In the next step, if $n=3$, the word $A_{1} A_{1}$ can be extended on $k$ possibilities and in each remaining $k(k-1)$ cases we can extend words on $(k-1)$ ways. Consequently, for $n=3$, we have $k+k(k-1)^{2}$ words. Proving analogously for fixed $n \geq 1$, we have exactly $k+(k-1)^{2}+(k-1)^{3}+\cdots+(k-1)^{n-2}+k(k-1)^{n-1}$ possibilities. By simple calculations, using the sum of the finite geometric series we have

$$
\begin{aligned}
k+(k-1)^{2}+ & (k-1)^{3}+\cdots+(k-1)^{n-2}+k(k-1)^{n-1} \\
& =k+(k-1)^{2} \frac{1-(k-1)^{n-3}}{1-(k-1)}+k(k-1)^{n-1} \\
& =k+\frac{1}{k-2}(k-1)^{2}\left((k-1)^{n-3}-1\right)+k(k-1)^{n-1} \\
& =k+\frac{1}{k-2}(k-1)^{n-1}-\frac{1}{k-2}(k-1)^{2}+k(k-1)^{n-1} \\
& =k+(k-1)^{n-1}\left(\frac{1}{k-2}+k\right)-\frac{1}{k-2}(k-1)^{2} \\
& =k+\frac{(k-1)^{n+1}}{k-2}-\frac{1}{k-2}(k-1)^{2} \\
& =\frac{1}{k-2}\left((k-1)^{n+1}-1\right)+\frac{1}{k-2}-\frac{(k-1)^{2}}{k-2}+k \\
& =\frac{1}{k-2}\left((k-1)^{n+1}-1\right),
\end{aligned}
$$

which ends the proof.

From the above and by the direct formula for the generalized Mersenne numbers, we have

Corollary 9. Let $n \geq 1, k \geq 3$ be integers. Then $\sigma(k, n)=M(k, n+1)$. 
Now we consider another combinatorial interpretation of the generalized Mersenne numbers $M(k, n)$, using given earlier alphabet $\mathcal{A}=\left\{A_{1}, \ldots, A_{k}\right\}$. For $k \geq 3$ and $n \geq 1$ we consider words $a_{1} a_{2} \ldots a_{n}$ of the length $n$ such that (iii) $a_{i} \neq A_{1}$ for all $i=1, \ldots, n$, or

(iv) $a_{j}=A_{1}$, for all $j=1, \ldots, p, 1 \leq p \leq n$ and $a_{i} \neq A_{1}$ for $p+1 \leq i \leq n$.

Let $\sigma^{*}(k, n)$ denote the number of all words $a_{1} \ldots a_{n}$ satisfying conditions (iii) and (iv).

Theorem 10. Let $n \geq 1, k \geq 3$ be integers. Then

$$
\sigma^{*}(k, n)=\frac{1}{k-2}\left((k-1)^{n+1}-1\right) .
$$

Proof. (By induction on $n$ ). If $n=1$, then $\sigma^{*}(k, 1)=k$ is obvious. Let $n \geq 2$ and suppose that the theorem is true for an arbitrary $n$. We shall show that it is true for $n+1$. By the induction hypothesis

$$
\sigma^{*}(k, n)=\frac{1}{k-2}\left((k-1)^{n+1}-1\right)=\left(\frac{1}{k-2}\left((k-1)^{n+1}-1\right)-1\right)+1,
$$

where the first summand denotes the number of words of the length $n$, where $a_{n} \neq A_{1}$ and the summand 1 corresponds to the word consisting only of $A_{1}$ i.e. the word $A_{1} A_{1} \ldots A_{1}$. Obviously, the word with $n+1$ letters can be obtained into $\left.\frac{1}{k-2}(k-1)\left((k-1)^{n}-1\right)-1\right)+k$ possibilities. Hence by simple calculations we get

$$
\begin{aligned}
\sigma^{*}(k, n) & =\frac{1}{k-2}\left((k-1)^{n+1}-k+1\right)-(k-1)+k \\
& =\frac{1}{k-2}\left((k-1)^{n+1}-k+1\right)+1=\frac{1}{k-2}\left((k-1)^{n+1}-1\right) \\
& =M(k, n+1),
\end{aligned}
$$

which ends the proof.

\section{REFERENCES}

[1] Berge, C., Principles of Combinatorics, Academic Press, New York-London, 1971.

[2] Civciv, H., Turkman, R., On the $(s, t)$-Fibonacci and Fibonacci Matrix Sequences, Ars Combin. 87 (2008), 161-173.

[3] Ercolano, J., Matrix generator of Pell sequences, Fibonacci Quart. 17 (1) (1979), $71-77$.

[4] Kaygisiz, K., Sahin, A., Determinant and permanent of the Hessenberg matrix and Fibonacci type numbers, Gen. Math. Notes 9 (2) (2012), 32-41.

[5] Kilic, E., On the usual Fibonacci and generalized order $k$-Pell sequences by Hessenberg matrices, Ars Combin. 94 (2010), 161-174.

[6] Kilic, E., Stanica, P., A matrix approach for general higher order linear recurrence, Bull. Malays. Math. Sci. Soc. (2) 34 (1) (2011), 51-67.

[7] Kilic, E., Tasci, D., On the generalized Fibonacci and Pell sequences by Hessenberg matrices, Ars Combin. 94 (2010) 161-174.

[8] Sergeer, A. S., Generalized Mersenne matrices and Balonin's conjecture, Automatic Control and Computer Sciences 48 (4) (2014), 214-220. 
[9] Solinas, J., Generalized Mersenne Numbers, Technical report CORR-39, Dept. of C. \& O., University of Waterloo, 1999.

Available from http://www.carc.math.uwaterloo.ca

[10] Włoch, A., Wołowiec-Musiał, M., Generalized Pell numbers and some relations with Fibonacci numbers, Ars Combin. 109 (2013), 391-403.

[11] Zheng, Y., Shon, S., Exact inverse matrices of Fermat and Mersenne circulant matrix, Abstr. Appl. Anal. 2015 (2015), Article 760823, 10 pp.

Paweł Ochalik

The Faculty of Mechanical Engineering and Aeronautics

Rzeszów University of Technology

al. Powstańców Warszawy 8

35-959 Rzeszów

Poland

e-mail: pawel.ochalik.96@gmail.com

Andrzej Włoch

The Faculty of Mathematics and Applied Physics

Rzeszów University of Technology

al. Powstańców Warszawy 8

35-959 Rzeszów

Poland

e-mail: awloch@prz.edu.pl

Received December 13, 2016 